\title{
The development of an innovative web based dietary assessment tool for an Irish adult population: The Diet Ireland tool
}

\author{
C.M. Timon 1 , K. Evans ${ }^{2}$, J. Walton ${ }^{2}$, A. Flynn ${ }^{2}$ and E.R. Gibney ${ }^{1}$ \\ ${ }^{1}$ Institute of Food and Health, University College Dublin, Belfield, Dublin 4, Ireland and ${ }^{2}$ School of Food and \\ Nutritional Sciences, University College Cork, Cork, Ireland.
}

Technological developments in recent years may offer solutions to the limitations of traditional dietary assessment methodologies (cost, participation rates and accuracy of data collected) (1). The aim of this project is to develop a web based 24-hour dietary recall tool for an Irish adult population.

The development of the tool involves 3 phases, 2 of which are already complete. Phase 1 used qualitative methods (surveys and interviews) to investigate the acceptability of the proposed tool. Respondents representing 9 stakeholder organisations/institutions in Ireland took part in a survey to evaluate the proposal of a web based dietary assessment tool. All of the respondents reported using dietary intake data for a multitude of different purposes but mainly to: inform nutrition and dietetic advice, develop educational and marketing material, assess effectiveness of nutritional intervention/strategy, conduct exposure assessment and assess brand/ food group intake data. Results highlighted that the majority of respondents were in favour of the development of a web based dietary assessment method and $75 \%$ of respondents stated they would use this type of tool in their respective organisation/institution. The respondents reported that the weighed food diary was their preferred method for collection of dietary intake data, but alternatively a 24 hour recall method was deemed preferential to a food frequency questionnaire or diet history. Findings from the stakeholder and literature analysis shaped the proposed design of the tool which follows the previously published (2) multi-pass 24 hour recall model, with in built linked foods and prompt questions to ensure complete capture of food intake data. The tool will also collect information on supplement use, occasionally consumed foods and eating behaviours.

Phase 2 involved the development of 3 comprehensive information databases (food and beverage list, nutritional composition data and portion size data) to populate the content of the Diet Ireland tool. This resulted in the compilation of a dataset of 926 food and beverage items (derived from the Irish National Adult Nutrition Survey) (3), with prompt questions proposed for 123 food and beverage items and linked food/beverage options for 255 foods. Phase 3 will involve the physical development of the software by Crème Global. On completion of Phase 3, the Diet Ireland tool will be used in comparative and validation studies to assess the acceptability and validity of the tool.

The project is funded by the Irish Department of Agriculture, Food and the Marine under the development of online dietary assessment tool, The Diet Ireland project $13 \mathrm{~F} 424$.

1. McCabe-Sellers B. Advancing the art and science of dietary assessment through technology (2010). J Am Diet Assoc 110(1):52-4..

2. Arab L, Wesseling-Perry K, Jardack P, Henry J, Winter A. Eight self-administered 24-hour dietary recalls using the Internet are feasible in African Americans and Whites: the energetics study (2010). J Am Diet Assoc 110(6):857-64

3. Irish Universities Nutrition Alliance. National Adult Nutrition Survey: Summary Report. Available from: http://www.iuna.net/wpcontent/uploads/ 2010/12/National-Adult-Nutrition-Survey-Summary-Report-March-2011.pdf 